\title{
Personality, Personal Model Beliefs, and Self-Care in Adolescents and Young Adults With Type 1 Diabetes
}

\section{T. Chas Skinner}

Department of Psychology University of Bath

Sarah E. Hampson

Department of Psychology University of Surrey

Chris Fife-Schaw

Department of Psychology University of Surrey

This paper appears as: Skinner, C.T., Hampson, S.E. \& Fife-Schaw, C.R. (2002). Personality, personal model beliefs, and self-care in adolescents and young adults with Type 1 diabetes.

Health Psychology, 21(1), 61-70.

\begin{abstract}
This study compared 3 models of association between personality, personal model beliefs, and self-care in a cross-sectional design. These models were as follows: (a) Emotional stability determines self-care indirectly through personal model beliefs, and conscientiousness is a direct predictor of self-care; (b) emotional stability determines selfcare indirectly through personal model beliefs, and conscientiousness moderates the association between beliefs and self-care; (c) both emotional stability and conscientiousness determine self-care indirectly through personal model beliefs. Participants ( $N=358$, aged 12 -30 years) with Type 1 diabetes completed measures of personality, personal model beliefs, and self-care. Structural equation modeling indicated that Model C was the best fit to the data.
\end{abstract}


One health-psychology model that is increasingly being used to understand and predict individuals' coping with and subsequent self-care of chronic illness is Leventhal and colleagues' self-regulation model ( Leventhal, Diefenbach, \& Leventhal, 1992 ; Leventhal, Nerenz, Steele, Taylor, \& Singer, 1984 ; Meyer, Leventhal, \& Gutmann, 1985 ). This model postulates that it is the individual's illness representation or personal model of illness that is the proximal determinant of coping behavior. In adults with diabetes, beliefs about the efficacy of their treatment and the perceived consequences and seriousness of diabetes were predictive of dietary and exercise self-management ( Hampson, Glasgow, \& Foster, 1995; Hampson, Glasgow, \& Toobert, 1990). In a sample of over 2,000 participants, beliefs about treatment effectiveness and the perceived seriousness of diabetes were predictive of self-care, with treatment effectiveness beliefs being a better predictor than barriers to adherence ( Glasgow, Strycker, Hampson, \& Ruggiero, 1997). In a prospective study of adolescents with diabetes, beliefs in the effectiveness of the treatment regimen were predictive of better dietary self-care ( Skinner \& Hampson, 1998 ; Skinner, John, \& Hampson, 2000 ). These prospective data also indicated that it was only perceived treatment effectiveness to control diabetes, not perceived treatment effectiveness to prevent the complications of diabetes, that was predictive of self-care. Therefore, in this study we hypothesized that beliefs about the perceived effectiveness of diabetes treatment would be a proximal predictor of self-care.

The role of personality in determining self-care behavior has been relatively ignored ( Van Heck, 1997; Wiebe \& Smith, 1997), possibly because of the lack of convergence in concepts and measures. However, with the emergence of the Big Five framework as the predominant model ( Digman, 1990; McCrae \& Costa, 1987), personality is receiving increased attention in health psychology. Two of the Big Five personality dimensions, emotional stability and conscientiousness, are seen as particularly relevant to health and health behavior.

Low scorers on emotional stability are characterized by the tendency to experience chronic negative emotions and to display related cognitive and behavioral characteristics ( Digman, 1990; McCrae \& Costa, 1987 ). Evidence does not support a direct association between emotional stability and health behavior ( Wiebe \& Smith, 1997 ). However, studies have consistently shown that greater emotional instability is associated with more frequent reporting of symptoms, reporting of greater severity of symptoms, more frequent contact with health care professionals, greater emotional distress, and poorer self-reported health ( Costa, Fleg, McCrae, \& Lakatta, 1982 ; Larsen, 1992 ; Shekelle, Vernon, \& Ostfeld, 1991 ). In patients with diabetes, lower emotional stability has been associated both with reporting of more symptoms when hypoglycemic and with greater worry about hypoglycemia ( Hepburn, Langan, Deary, \& Frier, 1994 ). Specifically, Hepburn and colleagues (1994) found that lower emotional stability was associated with greater worry about hypoglycemia, which in turn predicted hypoglycemia avoidance behavior. These findings suggest that emotional instability may be influencing individuals' perceptions of their bodily sensations, resulting in more negative illness beliefs and prognoses ( Christensen, Moran, \& Wiebe, 1999; Wiebe \& Smith, 1997 ). Therefore, we hypothesized that emotional stability would influence diabetes self-care behavior indirectly through its effect on illness beliefs (see Figure 1A).

Conscientiousness refers to traits such as reliability, perseverance, and self-discipline ( Digman, 1990; McCrae \& Costa, 1987 ), and there is increasing evidence that it is an important predictor of health behavior. In cross-sectional studies, conscientiousness has been associated with a wide range of behaviors ( Arthur \& Graziano, 1996; Booth-Kewley \& Vickers, 1994 ; Castle, Skinner, \& Hampson, 1999; Cox, Borger, Asmundson, \& Taylor, 
2000 ; Ingledew \& Brunning, 1999; Lemos-Giraldez \& Fidalgo-Aliste, 1997 ; Vollrath, Knoch, \& Cassano, 1999), with similar findings for prospective studies ( Friedman, Tucker, Schwartz, Martin, et al., 1995; Siegler, Feaganes, \& Rimer, 1995 ), including being predictive of greater longevity ( Friedman, Tucker, Schwartz, Tomlinson-Keasey, et al., 1995 ). In the context of chronic illness, conscientiousness has been associated with better self-care for those on renal dialysis ( Christensen \& Smith, 1995), better self-care in adults with type 1 diabetes ( Christensen et al., 1999), and longer renal deterioration times in individuals with type 1 diabetes ( Brickman, Yount, Blaney, Rothberg, \& De-Nour, 1996 ). The trait of conscientiousness overlaps substantially with the concept of ego strength or development used in pediatric research ( Friedman, Tucker, Schwartz, Martin, et al., 1995). Several studies have found that more mature ego development (impulse control, moral development, quality of interpersonal relations) is associated with better control of diabetes ( Barglow et al., 1983 ; Ryden, Nevander, Johnsson, Westbom, \& Sjoblad, 1990) and prospectively predictive of better metabolic control ( Jacobson et al., 1990).

Only a few studies have overtly examined the mechanism by which conscientiousness influences self-care behavior. Conscientiousness may act as an independent predictor of behavior. That is, highly conscientious people may generally act in a self-preserving way and be more likely to look after their health (see Figure 1A ). Alternatively, conscientiousness may moderate the association between information, knowledge, beliefs, and behavior. More conscientious individuals may be more likely to follow treatment recommendations and act on their knowledge of health risks and effectiveness of health behavior (see Figure 1B ). Schwartz and colleagues ( Schwartz et al., 1999) found support for the moderating effect of conscientiousness on distress and mammography utilization. Hampson and colleagues ( Hampson, Andrews, Barckley, Lichtenstein, \& Lee, 2000) found that perceived risk was predictive of reducing the number of cigarettes smoked indoors but only for those who were highly conscientious, which supported the moderating model. However, Wiebe and Christensen (1997) reported an interaction between conscientiousness and perceived severity in haemodyalysis patients, but contrary to predictions, individuals with high perceived risk and high conscientiousness had the poorest adherence.

Another possibility is that conscientiousness may be determining health behavior indirectly through illness beliefs (see Figure 1C). Vollrath et al. (1999) reported conscientiousness to be associated with preventive health behaviors both directly and indirectly by means of health beliefs. Similarly, Lemos-Giraldez and Fidalgo-Aliste (1997) reported that conscientiousness was associated both with health habits (e.g., smoking, alcohol consumption) and attitudes toward health. Christensen et al. (1999) reported a strong association between health beliefs and conscientiousness in adults with type 1 diabetes mellitus. The results of their regression analysis were suggestive of a partial mediating role of the health beliefs. That is, both conscientiousness and health beliefs were predictive of selfcare, and when health beliefs were entered into the regression the effect of conscientiousness on self-care was substantially reduced. However, because they were not explicitly testing a mediation model, they did not report whether conscientiousness was predictive of self-care, which is necessary to demonstrate a mediation effect ( Baron \& Kenny, 1986). Further support for this model comes from studies showing that conscientiousness is associated with a range of other health-relevant beliefs, such as locus of control, optimism, life purpose, and self-faith ( Marshall, Wortman, Vickers, Kusulas, \& Hervig, 1994 ), and with active problemfocused coping behavior ( Watson \& Hubbard, 1996 ). 
In this study, we set out to test these hypothesized associations among personality, personal models, and self-care in a sample of young adults and adolescents with type 1 diabetes. We developed three models from the hypothesized associations and included both emotional stability and conscientiousness in all three models so that we could examine the unique contributions of each personality trait. Therefore, in this study we set out to compare the following three models of association between personality, personal model beliefs, and self-care in a cross-sectional design: (a) Emotional stability determines self-care indirectly through beliefs about the consequences of diabetes, and conscientiousness is an independent predictor of self-care (see Figure 1A ); (b) emotional stability determines self-care indirectly through beliefs about the consequences of diabetes, and conscientiousness moderates the association between beliefs and self-care (see Figure 1B ); (c) both emotional stability and conscientiousness determine self-care indirectly through beliefs about the perceived consequences of diabetes and the perceived effectiveness of treatment, respectively (see Figure 1C).

\section{Method}

\section{Participants and Procedures}

Participants were all from the Young Diabetics (YD) category of membership of the British Diabetic Association for adolescents and young adults with diabetes. The questionnaire booklet was distributed along with a regular newsletter and an accompanying postage-paid envelope for returning the completed questionnaire. At the time of distribution, the YD membership was 1,276. However, those members who were resident overseas were excluded, resulting in a total of 1,200 questionnaires being distributed.

A total of 460 completed questionnaires were returned within 3 months, which was a $38 \%$ response rate. The vast majority (92\%) of respondents had type 1 diabetes mellitus, $4 \%$ reported having type 2 diabetes, and a further $4 \%$ did not respond to this question.

Respondents under 12 years were excluded from further analyses as were respondents with type 2 diabetes and those who reported having diabetes for less than 1 year. These exclusions reduced the sample to 406 respondents (34\% of those originally contacted) aged between 12 and 38 years. Any questionnaires with missing data were then excluded, because LISREL would not be able to analyze these cases, leaving a final sample of 338 cases. This final sample was not significantly different from the full sample on any demographic, personality, personal model belief, or self-care measure (see Table 1 ).

\section{Measures}

After the introductory letter on the front of the booklet, the first measure was the Summary of Diabetes Self-Care Activities ( Toobert \& Glasgow, 1994). This is a 12-item self-report instrument that assesses four areas of diabetes self-management (diet, exercise, blood glucose monitoring, and injecting - medication taking) over the previous 7 days. This is a wellvalidated instrument that has been widely used with both adult and adolescent samples ( Bond, Aiken, \& Somerville, 1992 ; Toobert, Hampson, \& Glasgow, 2000 ; Skinner \& Hampson, 1998 ). Five items ask about the amount and types of food eaten, three items address frequency of exercise, two items address frequency of blood glucose tests, and two items assess the frequency and timing of insulin injections. 
Responses to each item were standardized across the study sample ( Toobert \& Glasgow, 1994 ), and the mean standardized scores for each scale were calculated. The internal consistencies of the Diet, Exercise, and Blood Glucose Testing scales were adequate (greater than 0.70 , see Table 2 ); however, the internal consistency of the insulin administration scale was only 0.57 . All the self-care scales were moderately but significantly correlated (see Table 2), with a particularly strong correlation between Frequency of Blood Glucose Testing and Frequency of Exercise.

The second measure assessed personality by the Big Five Inventory 44 ( John, Donahue, \& Kentle, 1991 ), a 44-item questionnaire that assesses the Big Five personality dimensions of agreeableness, conscientiousness, extroversion, emotional stability, and openness to experience. Each item provides a self-descriptive statement, "I see myself as someone who ..." (is talkative; is a reliable worker; is sometimes shy; is inventive; can be tense), and participants rate the extent to which they agree or disagree with the statement on a 5-point scale ranging from 1 (disagree strongly) to 5 (agree strongly).

Because some of the respondents were younger than the validation samples used for the Big Five Inventory, we conducted a factor analysis on the personality items. Using principal-component analysis, with direct obliminal rotation, we imposed a five-factor solution on the data. The resulting solution accounted for $47 \%$ of the variance, with each item having a loading of greater than .4 on only one factor. All items loaded on the expected Big Five scales. We then calculated the scale scores according to the manual ( John et al., 1991). We computed the internal consistencies (Cronbach's alpha) of the scales and correlated the scales with each other. The Cronbach's alphas were all acceptable and similar to those found in the validation samples, with the exception of Openness to Experience, where the alpha was noticeably lower for this sample (.84) as compared with the validation sample ( John et al., 1991 ). The five scales were significantly (with one exception) but moderately correlated, with all correlations being below .30, indicating that the scales were relatively independent. The only nonsignificant correlation was between emotional stability and conscientiousness ( $r$ $=-.07)$. Table 3 gives the mean, standard deviation, and range of scores for these two scales. For emotional stability, these data suggested that there was no systematic bias in the group, with the mean score being just over the midpoint of the possible range for this scale and with the minimum and maximum scores covering almost the entire range of possible scores. Participants' mean scores also were not noticeably different from the population samples on which the instrument was developed. For conscientiousness, the mean scale score for the YD sample was 3.8 ( $S D=0.64)$; in the validation sample it was 3.6 ( $S D=0.65)$. For emotional stability, the YD sample's mean score was $3.2(S D=0.75)$ compared with 3.2 ( $S D$ $=0.76)$ in the validation sample ( John et al., 1991).

The third measure assessed two aspects of the participant's personal model of diabetes: the perceived consequences of diabetes and the perceived effectiveness of treatment. The 15-item Perceived Consequences scale was composed of the 10 items of the Perceived Consequences scale of the Illness Perception Questionnaire ( Weinman, Petrie, Moss-Morris, \& Horne, 1996 ; e.g., "My diabetes has strongly affected the way others see me") and a further two items derived from the Personal Models of Diabetes Interview ( Hampson et al., 1990, 1995; e.g., "I worry about getting the complications of diabetes"). There was a single item on fear of hypoglycemia, and there were two items asking about the threat of diabetes to the individual's current and future health. In response to each statement, participants were asked to rate their level of agreement on a 5-point rating scale ranging from 1 (strongly agree) to 5 ( strongly disagree). 
Perceived Treatment Effectiveness was assessed using two scales measuring effectiveness to control diabetes and effectiveness to prevent the complications of diabetes, which were based on scales developed by Glasgow and colleagues ( Glasgow et al., 1997). The Control scale asked participants to rate "How important is each of the following for controlling your diabetes" on a 5-point scale ranging from 1 ( not important) to 5 ( extremely important ). There were a total of eight items, two each on diet and blood glucose monitoring, with subsequent items on exercise, smoking, alcohol consumption, and managing sick days. The Prevention scale asked participants to rate "How likely is each of the following to help prevent complications of your diabetes" on a 5-point scale ranging from 1 ( not likely to help ) to 5 ( extremely likely to help). This scale had 10 items; the first 8 were the same as the scale on controlling diabetes and the additional two items concerned foot care and getting regular medical checks for diabetes-related complications. These two perceived treatment effectiveness scales were highly correlated but were retained as separate scales because previous studies indicated that these two constructs show different patterns of association with self-care behaviors. The perceived treatment effectiveness scales were relatively independent of the perceived consequences scales, with correlations below 0.20 , although three of these correlations were significant. From Table 2 , it can also be seen that there was a wide range in participants' responses, suggesting the full range of response options was used.

The last page of the questionnaire asked for demographic information (age, gender, employment status) and medical information (type of diabetes, duration, treatment regimen).

\section{Results}

\section{Sample Characteristics}

The demographic and medical information for this sample is summarized in Table 1 . There was a marked gender bias, with women being overrepresented. However, the YD membership is similarly biased, with just over $61 \%$ being female. Nevertheless, more women than men returned questionnaires, $\chi^{2}(1, N=388)=16.80, p<.001$. There were no significant gender differences on either age, $t(337)=-0.88, n s$, or duration of illness, $t$ $(337)=-0.19, n s$. There were no significant gender differences on injection regimen, $\chi^{2}(4$, $N=388)=1.10, n s$. However, male respondents were more likely to be students and less likely to be in a "professional" occupation, $\chi^{2}(6, N=388)=18.80, p<.005$.

\section{Measures}

Because the Perceived Consequences scale was derived from different instruments, the factor structure of the items required exploration. Because there was no hypothesized factor structure, we randomly split the sample into two groups so that we could conduct exploratory analyses and confirmatory analyses on separate samples. The first group's responses were factor analyzed using principal-components analyses with direct obliminal rotation. Factor selection was based on eigenvalues greater than 1 . These analyses resulted in a three-factor solution, which accounted for $50 \%$ of the variance. However, the third factor had only one item with a loading greater than 0.40 . Therefore, we repeated the analysis on the same subsample with a forced two-factor solution. The two factors identified accounted for $43 \%$ of the variance. No item had a loading of greater than 0.40 on both factors, with one item not loading on either scale. 
The data for the second half of the sample were similarly factor analyzed, using an imposed two-factor solution. The two identified factors accounted for $44 \%$ of the variance. No item loaded greater than .4 on both factors. However, this analysis indicated that two additional items did not load on either factor, and so in total three items were dropped from the measures. Finally, comparison of the two analyses indicated that one item loaded on different factors in each analysis, and this item was also excluded.

For the two factors we identified, both had items that loaded greater than 0.40 on only one factor, both had a loading that was consistent across subsamples, and for both the items within the factor had theoretical coherence. Therefore these factors met the criteria for simple structure, and we computed scale scores using the mean item response. Six items loaded on the first factor, which was labeled Perceived Impact ("My diabetes has: not had much effect on my life (negative); strongly affected my family; strongly affected the way I see myself as a person; strongly affected the way others see me; changed my daily activities; means I have less independence"). The second factor was labeled Perceived Threat of Diabetes and consisted of five items ("My diabetes is serious; My diabetes is a serious threat to my future health; My diabetes is a serious threat to my current health; I worry about getting the complications of diabetes; I will probably get diabetes complications"). As can be seen from $\underline{\text { Table } 2}$, the internal consistency of these scales (Cronbach's alphas) was adequate, being above .74, and the two scales were moderately correlated.

\section{Demographic Effects}

There were only two significant gender effects after controlling for multiple comparisons. Women described themselves as more emotionally unstable ( $M=3.35, S D=0.73)$ than men $(M=2.96, S D=0.74), t(399)=4.62, p<.0001$, and women reported that their treatment was more likely to prevent complications ( $M=4.00, S D=0.6)$ than men $(M=3.81, S D=$ $0.6), t(396)=2.80, p<.005$. Older participants were more likely to be in a higher socioeconomic group ( $r=-.27, n=184, p<.001$ ), they described themselves as more conscientious ( $r=.18, n=400, p<.0001)$, they reported that diabetes had a greater impact on their life $(r=-.18, n=403, p<.001)$, and they reported that diabetes was a greater threat to their health $(r=-.18, n=402, p<.001)$. Neither duration of illness nor socioeconomic status was associated with any personality, personal model beliefs, or selfcare measure.

\section{Bivariate Analyses}

Table 2 shows the associations between personal model beliefs, personality, and self-care. Consistent with the hypothesized models, perceived treatment effectiveness to control diabetes and perceived treatment effectiveness to prevent complications were significantly associated with self-care. For all four self-care measures, effectiveness to control diabetes showed noticeably stronger associations. Perceived threat of diabetes was similarly associated with three self-care measures (blood glucose testing being the exception), with greater threat associated with better self-care. The perceived impact of diabetes was not associated with any of the self-care measures.

As hypothesized, less emotional stability was associated with the greater perceived threat and greater perceived impact of diabetes. In order for treatment effectiveness beliefs to mediate the association between self-care and conscientiousness, all three variables must be associated, which is evident in Table 2 . Conscientiousness was associated with greater 
perceptions in the efficacy of treatment-but not with beliefs about impact or threat of diabetes-and was also associated with all four self-care measures.

\section{Model Testing}

To test the three competing models, we used structural equation modeling (SEM). In SEM, the researcher proposes a theoretically derived model (or set of models in this case) of the relationships between variables, and the SEM program generates a covariance matrix that is implied by the model. For a model to gain support, the covariance matrix implied by the model should be little different from the observed covariance matrix. A number of indices exist to assist in determining how well the model-implied and observed matrices fit each other. These indices are conventionally interpreted in combination rather than relying on a single index. In the present study, we looked for a nonsignificant goodness-of-fit chi-square, a root mean square error of approximation (RMSEA) of less than .05, a normed fit index (NFI) of greater than .95, and an adjusted goodness of fit index (AGFI) greater than .95. In addition to the model being a good fit to the data, the individual paths in the model must be statistically significant and theoretically meaningful. All analyses were conducted using LISREL 8.30.

For Personal Model Beliefs, the measurement error entered for each of these variables was set at one minus the internal consistency of the scales multiplied by the scale variance. For the personality measures, the internal consistency of Emotional Stability was the same in both the validation studies ( John et al., 1991) and data reported here. However, for Conscientiousness, the internal consistency of the scale reported by John and colleagues (1991) was marginally lower than for this sample and was therefore used in this analysis to calculate the error terms. For Self-Care, the measurement error entered for these scales was based on the internal consistency data reported here.

\section{Model 1 ( Figure 1A ).}

To test this model, we entered perceived threat, perceived impact, perceived treatment effectiveness to control, and perceived treatment effectiveness to prevent complications as determinants of self-care. Conscientiousness was entered as a direct independent predictor of all self-care measures. Emotional stability was entered as a determinant of personal model beliefs, and all self-care measures were assumed to be intercorrelated.

Although the chi- square was significant, other indices indicated that this model was approaching an adequate fit, $\chi^{2}(18, N=388)=32.79, p=.018($ RMSEA $=0.05$; NFI $=90$; AGFI $=.93$ ). Path coefficients indicated that emotional stability was not a significant determinant of either perceived treatment effectiveness beliefs, but it was a significant predictor of perceived threat to health and perceived impact. Perceived impact was not predictive of any self-care measure, and the paths between diet and the other three self-care measures were not significant. Therefore, we removed these paths from all subsequent analyses. We then retested this model, which showed an improved fit, $\chi^{2}(16, N=388)=$ $13.20, p=.64 ; \Delta \chi^{2}(1, \mathrm{~N}=388)=19.59, p \sim 0(\mathrm{RMSEA}=0.001 ; \mathrm{NFI}=.96 ; \mathrm{AGFI}=.96)$. However, examination of the $t$ value for each path indicated that the paths from conscientiousness to insulin administration, blood glucose testing, and exercise were all nonsignificant. Because this model was testing a direct effect of personality on self-care, the fact that these paths were all nonsignificant indicated that this model was not supported by the analysis. 


\section{Model 2 ( Figure 1B ).}

To test this model, we computed four cross-product interaction terms and error variances (variables centered then multiplied) ${ }^{1}$ between perceived impact and perceived threat with conscientiousness and both perceived treatment effectiveness measures and conscientiousness. We then retested the moderator model by having conscientiousness, perceived threat, perceived treatment effectiveness to control diabetes, perceived treatment effectiveness to prevent complications, and all interaction terms as predictors of self-care.

The results indicated that this was not a good fit to the data, because the chi-square was significant, $\chi^{2}(28, N=388)=34.85, p<.0005$, RMSEA was greater than 0.07 (NFI = .96; AGFI .91), and none of the paths from the interaction terms to self-care measures was significant. We repeated the analyses without the interaction terms for perceived threat and impact, given that these were not associated with conscientiousness, but there was no noticeable improvement in fit indices. Therefore the moderator model at no time proved to adequately fit the data.

\section{Model 3 ( Figure 1C).}

We next tested the mediation model with conscientiousness acting as a determinant of perceived impact, perceived threat, and both perceived treatment effectiveness measures. Both perceived treatment effectiveness measures and perceived threat were entered as determinants of self-care (perceived impact was not entered as a predictor of self-care, given the results of Model 1). This model also provided a good fit, $\chi^{2}(20, N=388)=20.25, p=$ $.33($ RMSEA $=0.02 ; \mathrm{NFI}=0.95 ; \mathrm{AGFI}=0.98)$. However, the paths from conscientiousness to perceived threat and perceived impact were not significant. Perceived threat was a nonsignificant predictor of diet; perceived treatment effectiveness to prevent complications was a nonsignificant predictor of exercise, blood glucose testing, and insulin use; and perceived impact was not predictive of any self-care measure. Perceived treatment effectiveness to control diabetes was a significant predictor of all self-care measures. Therefore, we repeated this analysis removing these nonsignificant paths. These changes did not improve the fit indices; however, all paths in the model were significant and the model was simplified, $\chi^{2}(26, N=388)=30.43, p=.25(\mathrm{RMSEA}=0.02 ; \mathrm{AGFI}=0.96$; NFI $=$ 0.95). This finding indicated that the model was a good fit to the data, and because the paths from conscientiousness to both perceived treatment effectiveness beliefs were significant, along with the paths from emotional stability to perceived threat and impact, this model was supported.

Given these results, the only model that provided an adequate fit to the data and had significant paths from conscientiousness to self-care (by means of perceived treatment effectiveness) was the mediation model ( Figure 1c ). That is, conscientiousness influences self-care indirectly through its influence on treatment effectiveness beliefs, and emotional stability influences self-care indirectly through its influence on perceived threat.

\section{Final Model}

As a last step in the model building, we entered demographic effects into the model on the basis of the results of the correlations in Table 2 . Gender was entered as a determinant of emotional stability and perceived treatment effectiveness to prevent complications. Age was entered as a determinant of conscientiousness, perceived impact, and perceived threat. The 
LISREL analysis suggested fit would be improved by adding a direct path from age to dietary self-care. This final model was tested and proved to be a good fit to the data, $\chi^{2}(41, N=388)$ $=49.66, p=.17(\mathrm{RMSEA}=0.025 ; \mathrm{NFI}=.93 ; \mathrm{GFI}=.98 ; \mathrm{AGFI}=.95)$, with all paths significant (see Figure 2 ). These additions indicated that women described themselves as less emotionally stable and described perceived treatment to be more effective in preventing complications than men. Older individuals were more conscientious, had better dietary selfcare, and thought that the diabetes was a greater threat to their health and had a greater impact on their life.

\section{Discussion}

The most important findings of this study emerged from the comparison of the different models linking personality, personal model beliefs, and self-care. The results of the modeling indicated that the effects of personality on self-care were mediated by beliefs. These effects were specific for each personality trait. The effects of emotional stability on self-care were mediated through its influence on beliefs about the threat of diabetes to individuals' health. In comparison, conscientiousness evidenced strong consistent associations with self-care, but these associations were mediated by beliefs about the effectiveness of their treatment regimen and not beliefs about the threat of diabetes.

The results of the SEM indicated that perceived treatment effectiveness to control diabetes is predictive of all aspect of diabetes self-care. In comparison, perceived treatment effectiveness to prevent the complications of diabetes was predictive only of frequency of blood glucose testing. This finding replicates the results of a 1-year prospective study of adolescents with diabetes, which reported perceived treatment effectiveness to prevent complications was not predictive of any aspect of self-care, whereas perceived treatment effectiveness to control diabetes was associated with better self-care ( Skinner \& Hampson, 1998 ; Skinner et al., 2000 ). Furthermore, change in beliefs about treatment effectiveness to control diabetes predicted change in self-care ( Skinner \& Hampson, 2001 ).

These results suggest the need to distinguish between short- and long-term treatment effectiveness beliefs. Short-term beliefs about treatment effectiveness may be more consistent with the time frame of patients' goal setting, especially in a population as young as the one studied here. However, this time frame of beliefs is also related to the nature of the goals. Perceived treatment effectiveness to control diabetes is about achieving a positive outcome (better control of diabetes, which is associated with a better quality of life), whereas perceived treatment effectiveness to prevent complications is about the avoidance of experiencing a negative outcome (complications). These two issues are clearly confounded in this study, and they need separating in future studies.

An additional point to note is that the factor analysis of the Perceived Consequences scale items suggested that there are two different dimensions to this aspect of an individual's personal model of diabetes: the impact that diabetes has on the individual and the threat of diabetes to the individual's health. This distinction between impact and threat is important because these two dimensions seem to relate to two different facets of adjustment to diabetes. Perceived impact was unrelated to self-care in this study, replicating the results of a prospective study of personal model beliefs of adolescents with diabetes ( $\underline{\text { Skinner \& }}$ Hampson, 1998 ; Skinner et al., 2000 ). However, perceived impact may be a key determinant of the individual's emotional response to living with diabetes ( Talbot, Nouwen, Gingras, Belanger, \& Audet, 1999), with changes in perceived impact associated with changes in 
adolescents' well-being ( Skinner \& Hampson, 1998 ; Skinner et al., 2000 ). In contrast, the threat of diabetes to the individual's health was associated with self-care behavior, replicating previous research using constructs from the health belief model ( Bond et al., 1992; Brownlee-Duffeck et al., 1987) and protection motivation theory ( Palardy, Greening, Ott, Holderby, \& Atchison, 1998 ).

The results presented here have some important implications for personality research. First, emotional stability was associated only with beliefs about the consequences of diabetes, not with beliefs about the effectiveness of treatment. Using a cold inoculation paradigm, Feldman and colleagues ( Feldman, Cohen, Doyle, Skoner, \& Gwaltney, 1999) suggested that the effects of emotional stability may operate through attentional processes. They suggested that emotional stability's effects are a result of the tendency for the less emotional stable person to be more self-focused and attentive to his or her physical state. The Perceived Consequences Scales are inherently self-focused (i.e., diabetes' impact on and threat to the self). In addition, emotional stability may exaggerate negative appraisal processes. Because both impact and threat are appraisals of negative outcomes, emotional stability will affect these cognitions. In contrast, treatment effectiveness beliefs are behavior-focused, not selffocused, and as appraisals of outcome expectancies, they may be positive or negative. Consequently, treatment effectiveness beliefs are less likely to be related to emotional stability.

Researchers have frequently emphasized that health behavior often requires the person to delay gratification in order to obtain advantageous health results, and the trait of conscientiousness is likely to be associated with such a willingness to delay gratification ( Booth-Kewley \& Vickers, 1994 ; Lemos-Giraldez \& Fidalgo-Aliste, 1997 ). However, the lack of a direct effect of conscientiousness on self-care, and the finding that conscientiousness was related to both short- and long-term treatment effectiveness beliefs, suggests that other processes may be driving the association between conscientiousness and health behavior. Examination of research on coping and personality suggests at least one plausible mechanism for the mediating role of treatment effectiveness beliefs on the association between conscientiousness and self-care. In particular, conscientiousness has been shown to be related to active, problem-focused coping styles ( Watson \& Hubbard, 1996 ). This finding suggests that in response to the stress of diabetes, more conscientious individuals seek out more information about their diabetes and its management, which in turn may result in stronger beliefs in the effectiveness of treatment. Conscientiousness has also been associated with optimistic control ( Marshall et al., 1994 ), suggesting that highly conscientious individuals may have a more optimistic view of their treatment and its benefit. Whether these or other mechanisms are responsible for the association between conscientiousness and treatment effectiveness beliefs clearly warrants further investigation.

The fact that the analysis reported here did not support a moderating role of conscientiousness on the association between personal model beliefs and self-care was surprising, given previous research findings ( Hampson, Andrews, et al., 2000 ; Schwartz et al., 1999). However, both of those previous studies considered preventive health behavior in ostensibly healthy individuals. In comparison, both the study by Wiebe and Christensen (1997), who found a counterintuitive moderator effect for conscientiousness, and the present study were conducted on individuals with a serious chronic illness. The experience of living with a chronic illness places the beliefs in a very different context. The possibility of different mechanisms operating in preventive health behavior, when anticipating a future health threat, 
versus illness self-management, when responding to a current health threat, demands further investigation.

The fact that personal model beliefs mediated the association between personality and self-care has important clinical implications. The more proximal role of personal model beliefs suggests that interventions need not target the individual's personality. Rather, interventions focusing on patient beliefs, which are more malleable than personality, should be effective, as demonstrated by the growing literature on cognitive-behavioral approaches to diabetes educational and psychosocial interventions ( van der Ven, Chatrou, \& Snoek, 2000 ).

This study has limitations. The sample cannot be considered fully representative of young British people with type 1 diabetes mellitus because there was overrepresentation of women, persons from higher socioeconomic status occupational groups, and probably Caucasian individuals. However, it should be noted the sample means suggest that this sample was not an overly conscientiousness group. The cross-sectional nature of the data also limits the casual inferences that can be drawn. Some consideration should also be given to the wording of the perceived treatment effectiveness scales. The Control scale asked how "important" each behavior is, whereas the Prevention scale asked how "likely" each behavior was to help. This distinction in terminology means that the two scales confound different time frames with different semantics. Future work should resolve this issue before the importance of short-term beliefs can be fully substantiated. However, these analyses provide an initial model to test on a more representative sample of young people with diabetes. Ideally such a future study should also be prospective in order to overcome the inherent problems of making causal inferences from cross-sectional data.

Despite these weaknesses, the data presented here and elsewhere ( Hampson et al., 1990, 1995; Glasgow et al., 1997; Skinner \& Hampson, 1998, 2001 ; Skinner, White, Johnston, \& Hixenbaugh, 1999) indicate that personal model beliefs are proximal determinants of self-care and it is time to develop interventions designed to target these beliefs. In addition, this study suggests that personality plays an indirect and distal role in self-care. It could be argued that some of the interventions already developed or being developed in the literature may be explicitly or implicitly targeting personal models of diabetes, such as blood glucose awareness training ( Gonder-Frederick, Cox, Clarke, \& Julian, 2000) and group cognitive behavior therapy ( van der Ven et al., 2000). Therefore, assessing the impact of these educational programs on personal model beliefs and subsequent self-care and well-being would be an efficient means of assessing the proximal role of these beliefs.

Although personality is not implicated as a target for intervention, it may be that personality measures can be used to assess the need for educational or psychological input. For example, it has been argued that patients with high scores on conscientiousness, predominantly because of better self-care, profit more from brief interventions directed at learning self-regulation skills ( Muten, 1991). Patients with low scores on emotional stability may benefit from more therapeutic, versus educational, input to address their elevated concerns about the consequences of diabetes and subsequent negative affect. 


\section{References}

Arthur, W. J. \& Graziano, W. G. (1996). The five-factor model, conscientiousness and driving accident involvement. Journal of Personality, 64, 593-618.P

Barglow, P., Edidin, D. V., Budlong-Springer, A. S., Berndt, D., Phillips, R. \& Dubow, E. (1983). Diabetic control in children and adolescents: Psychcosocial factors and therapeutic efficacy. Journal of Youth and Adolescence, 12, 77-94.

Baron, R. M. \& Kenny, D. A. (1986). The moderator-mediator variable distinction in social psychology research: Conceptual, strategic and statistical considerations. Journal of Personality and Social Psychology, 51, 1173-1182. P

Bond, G. G., Aiken, L. S. \& Somerville, S. C. (1992). The health belief model and adolescents with insulin-dependent diabetes mellitus. Health Psychology, 11, 190-198. $\mathrm{P}$ Booth-Kewley, S. \& Vickers, R. R. (1994). Associations between major domains of personality and health behavior. Journal of Personality, 62, 281-298.

Brickman, A. L., Yount, S. E., Blaney, N. T., Rothberg, S. T. \& De-Nour, A. K. (1996).

Personality traits and long-term health status: The influence of neuroticism and conscientiousness on renal deterioration in type-1 diabetes. Psychosomatics, 37, 459-468. P Brownlee-Duffeck, M., Peterson, L., Simonds, J. F., Goldstein, D., Kilo, C. \& Hoette, S. (1987). The role of health beliefs in the regimen adherence and metabolic control of adolescents with diabetes mellitus. Journal of Consulting and Clinical Psychology, 55, 139144. P

Castle, C. M., Skinner, T. C. \& Hampson, S. E. (1999). Young women and suntanning: An evaluation of a health education leaflet.(Psychology and Health, 14, 517-527.)

Christensen, A. J., Moran, P. J. \& Wiebe, J. S. (1999). Assessment of irrational health beliefs: Relation to health practices and medical regimen adherence. Health Psychology, 18, 169-176.

Christensen, A. J. \& Smith, T. W. (1995). Personality and patient adherence: Correlates of five-factor model in renal dialysis. Journal of Behavioral Medicine, 18, 305-313. Costa, P. T., Fleg, J. L., McCrae, R. R. \& Lakatta, E. G. (1982). Neuroticism, coronary artery disease, and chest pain complaints. Cross-sectional and longitudinal studies. Experimental Ageing Research, 8, 37-44.

Cox, B. J., Borger, S. C., Asmundson, G. J. G. \& Taylor, S. (2000). Dimensions of hypochondrias and the five-factor model of personality. Personality and Individual Differences, 29, 99-108.

Digman, J. M. (1990). Personality structure: Emergence of the five-factor model. Annual Review of Psychology, 41, 417-440.P

Feldman, P. J., Cohen, S., Doyle, W. J., Skoner, D. P. \& Gwaltney, J. M. (1999). The impact of personality on the reporting of unfound symptoms and illness. Journal of Personality and Social Psychology, 77, 370-378. P

Friedman, H. S., Tucker, J. S., Schwartz, J. E., Martin, L. R., Tomlinson-Keasey, C., Wingard, D. L. \& Criqui, M. H. (1995). Childhood conscientiousness and longevity: Health behaviors and cause of death. Journal of Personality and Social Psychology, 68, 696-703.P Friedman, H. S., Tucker, J. S., Schwartz, J. S., Tomlinson-Keasey, C., Martin, L. R., Wingard, D. L. \& Criqui, M. H. (1995). Psychosocial and behavioral predictors of longevity: The aging and death of the "Termites." American Psychologist, 50, 69-78. $\mathrm{P}$

Glasgow, R. E., Strycker, L. A., Hampson, S. E. \& Ruggiero, L. (1997). Personal-model beliefs and social environmental barriers related to diabetes self-management. Diabetes Care, 20, 556-561.

Gonder-Frederick, L., Cox, D., Clarke, W. \& Julian, D. (2000). Blood glucose awareness 
training.(In F. J. Snoek \& T. C. Skinner (Eds.), Psychology in diabetes care (pp. 169-208). Chichester, England: Wiley.)

Hampson, S. E., Andrews, J. A., Barckley, M., Lichtenstein, E. \& Lee, M. E. (2000).

Conscientiousness, perceived risk and risk-reduction behaviors: A preliminary study. Health Psychology, 19, 496-500. P

Hampson, S. E., Glasgow, R. E. \& Foster, L. S. (1995). Personal models of diabetes among older adults: Relationship to self-management and other variables. The Diabetes Educator, 21, 300-307.

Hampson, S. E., Glasgow, R. E. \& Toobert, D. J. (1990). Personal models of diabetes and their relations to self-care activities. Health Psychology, 9, 632-646. P

Hepburn, D. A., Langan, L. J., Deary, K. M. \& Frier, B. M. (1994). Psychological and demographic correlates of glycaemic control in adult patients with type I diabetes. Diabetic Medicine, 11, 578-582.

Ingledew, D. K. \& Brunning, S. (1999). Personality, preventive behavior and comparative optimism about health problems. Journal of Health Psychology, 4, 193-208.

Jacobson, A. M., Hauser, S. T., Lavori, P., Wolfsdorf, J. I., Herskowitz, R. D., Milley, J. E., Bliss, R., Gelfand, E., Wertileb, D. \& Stein, J. (1990). Adherence among children and adolescents with insulin-dependent diabetes mellitus over a four year longitudinal follow-up: 1. The influence of patient coping and adjustment. Journal of Pediatric Psychology, 15, 511526. P

John, O. P., Donahue, E. M. \& Kentle, R. L. (1991). The Big Five Inventory: Versions 4a and 54. Technical report. (Berkeley: University of California)

Larsen, R. J. (1992). Neuroticism and selective encoding and recall of symptoms: Evidence from a combined concurrent - retrospective study. Journal of Personality and Social Psychology, 62, 480-488. P

Lemos-Giraldez, S. \& Fidalgo-Aliste, A. M. (1997). Personality dispositions and healthrelated habits and attitudes: A cross sectional study. European Journal of Personality, 11, 197-209. P

Leventhal, H., Diefenbach, M. \& Leventhal, E. A. (1992). Illness cognitions: Using common sense to understand treatment adherence and affect cognition interactions. Cognitive Therapy and Research, 16, 143-163.

Leventhal, H., Nerenz, D. R., Steele, D. J., Taylor, S. E. \& Singer, J. E. (1984). Illness representation and coping with health threats.(In A. Baum (Ed.), Handbook of psychology and health (pp. 219-252). Hillsdale, NJ: Erlbaum.)

Marshall, G. N., Wortman, C. B., Vickers, R. R., Kusulas, J. W. \& Hervig, L. K. (1994). The five-factor model of personality as a framework for personality-health research. Journal of Personality and Social Psychology, 67, 278-286.

McCrae, R. R. \& Costa, P. T. (1987). Validation of the five-factor model of personality across instruments and observers. Journal of Personality and Social Psychology, 52, 81-90.P Meyer, D., Leventhal, H. \& Gutmann, M. (1985). Common-sense models of illness: The example of hypertension. Health Psychology, 4, 115-135.

Muten, E. (1991). Self-reports, spouse ratings and psychophysiological assessment in a behavioral medicine program: An application of the five-factor model. Journal of Personality Assessment, 57, 449-464. P

Palardy, N., Greening, L., Ott, J., Holderby, A. \& Atchison, J. (1998). Adolescents' health attitudes and adherence to treatment for insulin-dependent diabetes mellitus. Developmental and Behavioral Pediatrics, 19, 31-37.P

Ryden, O., Nevander, L., Johnsson, P., Westbom, L. \& Sjoblad, S. (1990). Diabetic children and their parents: Personality correlates of metabolic control. Acta Paeiatricia Scandanavia, $79,1202-1212$. 
Schwartz, M. D., Taylor, K. L., Willard, K. S., Siegel, J., Lamdam, R. M. \& Moran, K. (1999). Distress, personality and mammography utilization among women with a family history of breast cancer. Health Psychology, 18, 327-332. $\mathrm{P}$

Shekelle, R. B., Vernon, S. W. \& Ostfeld, A. M. (1991). Personality and coronary artery disease. Psychosomatic Medicine, 43, 117-125.P

Siegler, I. C., Feaganes, J. R. \& Rimer, B. K. (1995). Predictors of adoption of mammography in women under age 30. Health Psychology, 14, 274-278. P

Skinner, T. C. \& Hampson, S. E. (1998). Social support and personal models of diabetes in relation to self-care and well-being in adolescents with type 1 diabetes mellitus. Journal of Adolescence, 21, 703-715. P

Skinner, T. C. \& Hampson, S. E. (2001). Personal models of diabetes in relation to self-care and well-being, and glycemic control. A prospective study in adolescence. Diabetes Care, 24, 828-833.

Skinner, T. C., John, M. \& Hampson, S. E. (2000). Social support and personal models of diabetes as predictors of self-care and well-being: A longitudinal study of adolescents with diabetes. Journal of Pediatric Psychology, 25, 257-268. P

Skinner, T. C., White, J., Johnston, C. \& Hixenbaugh, P. (1999). Interaction between social support and injection regimen in predicting teenagers' concurrent glycosylated haemoglobin assays. Journal of Diabetes Nursing, 3, 140-144.

Talbot, F., Nouwen, A., Gingras, J., Belanger, A. \& Audet, J. (1999). Relations of diabetes intrusiveness and personal control to symptoms of depression among adults with diabetes.

Health Psychology, 18, 537-542.

Toobert, D. J. \& Glasgow, R. E. (1994). Assessing diabetes self-management: The Summary of Diabetes Self-Care Activities Questionnaire.(In C. Bradley (Ed.), Handbook of psychology and diabetes (pp. 351-374). Chur, Switzerland: Harwood Academic Publishers.)

Toobert, D. J., Hampson, S. E. \& Glasgow, R. E. (2000). The Summary of Diabetes SelfCare Activities Measure. Diabetes Care, 23, 943-950.

van der Ven, N. C. W., Chatrou, M. \& Snoek, F. J. (2000). Cognitive behavioural group training.(In F. J. Snoek \& T. C. Skinner (Eds.), Psychology in diabetes care (pp. 207-234). Chichester, England: Wiley.)

Van Heck, G. L. (1997). Personality and physical health: Toward an ecological approach to health-related personality research. European Journal of Personality, 11, 415-443.

Vollrath, M., Knoch, D. \& Cassano, L. (1999). Personality, risky health behaviour, and perceived susceptibility to health risks. European Journal of Personality, 13, 39-50. 8 Watson, D. \& Hubbard, B. (1996). Adaptational style and dispositional structure: Coping in the context of the five-factor model. Journal of Personality, 64, 737-774.P

Weinman, J., Petrie, K. J., Moss-Morris, R. \& Horne, R. (1996). The Illness Perception Questionnaire: A new method for assessing the cognitive representation of illness.

Psychology and Health, 11, 431-445.P

Wiebe, D. J. \& Smith, T. W. (1997). Personality and health: Progress and problems in psychosomatics.(In R. Hogan, J. Johnson, \& S. Briggs (Eds.), Handbook of personality psychology (pp. 891-918). London: Academic Press.)

Wiebe, J. S. \& Christensen, A. J. (1997). Health beliefs, personality and adherence in hemodialysis patients: An interactional perspective. Annals of Behavioral Medicine, 19, 3035. 


\section{Footnote:}

1

The error variance was calculated by generating two split half scores for each scale. These were then used to generate two product interaction terms for each interaction in the analysis. These two interaction terms were then correlated partialing out the effects of the linear terms (i.e., the split half scales) and adjusted using the Spearman-Brown formula. The error variance was then set at 1 - this reliability.

Correspondence may be addressed to T. Chas Skinner, Department of Psychology, University of Bath, Bath BA2 7AY, United Kingdom,

Electronic mail may be sent to t.c.skinner@bath.ac.uk 
Table 1. Demographics and Medical Details of Respondents

Table 1

Demographics and Medical Details of Respondents

\begin{tabular}{|c|c|c|c|c|c|c|c|c|c|c|c|}
\hline \multirow[b]{3}{*}{ Participant } & \multirow{2}{*}{\multicolumn{2}{|c|}{$\begin{array}{c}\text { Age } \\
\text { (years) }\end{array}$}} & \multirow{2}{*}{\multicolumn{2}{|c|}{$\begin{array}{l}\text { Duration } \\
\text { (years) }\end{array}$}} & \multirow{2}{*}{\multicolumn{3}{|c|}{ Regimen (\%) }} & \multicolumn{4}{|c|}{ SES $(\%)$} \\
\hline & & & & & & & & \multirow{2}{*}{$\begin{array}{c}\text { FT } \\
\text { student }\end{array}$} & \multirow[b]{2}{*}{ Pro-Int } & \multirow{2}{*}{$\begin{array}{l}\text { Skilled } \\
\text { worker }\end{array}$} & \multirow{2}{*}{$\begin{array}{l}\text { Semi- or } \\
\text { unskilled } \\
\text { worker }\end{array}$} \\
\hline & M & $S D$ & M & $S D$ & $2^{a}$ & $4^{2}$ & Other & & & & \\
\hline Men" & 22.2 & 4.4 & 10.7 & 6.7 & 32 & 6] & 7 & 43 & 17 & 24 & 4 \\
\hline Women ${ }^{e}$ & 21.8 & 4.1 & 10.6 & 6.1 & 35 & 58 & 7 & 35 & 20 & 24 & 6 \\
\hline Missing data & 21.7 & 4.1 & 10.5 & 5.9 & 36 & 58 & 6 & 44 & 17 & 22 & 3 \\
\hline
\end{tabular}

Note. SES $=$ socioeconomic status; $\mathrm{FT}=$ full-time; Pro $=$ professional; Int $=$ intermediate.

${ }^{2}$ Injections per day, ${ }^{b} n-95 ; 28 \%$. ${ }^{n} n=243 ; 72 \%$.

Table 2. Cronbach's Alpha for all Scales and Correlations Between Personality, Personal Model Beliefs, and Self-Care

Table 2

Cronbach's Alpha for all Scales and Correlations Between Personality, Personal Model Beliefs, and Self-Care

\begin{tabular}{|c|c|c|c|c|c|c|c|c|c|c|}
\hline Variable & Alpha & 1 & 2 & 3 & 4 & 5 & 6 & 7 & 8 & 9 \\
\hline 1. Diet & .71 & & & & & & & & & \\
\hline 2. Exercise & .85 & $.32 \% 8$ & & & & & & & & \\
\hline 3. Blood glucose testing & .82 & $.27^{* 06}$ & $59^{\mathrm{k*}}$ & & & & & & & \\
\hline 4. Insulin administration & .57 & $34=$ & $.22^{* *}$ & $.19^{* *}$ & & & & & & \\
\hline 5. Perceived impact & .80 & .07 & .02 & -.03 & .09 & & & & & \\
\hline 6. Perceived threat & .78 & $.23^{88}$ & $.18^{* *}$ & $.12^{*}$ & $.23 * *$ & $.46^{8 *}$ & & & & \\
\hline 7. Effectiveness to control diabetes & .74 & $32^{5 \pi}$ & $.31^{\text {** }}$ & $33^{\text {ta }}$ & $.20^{\circ \rightarrow}$ & $-.11^{*}$ & -.10 & & & \\
\hline 8. Effectiveness to prevent complications & .82 & $.17^{* \infty}$ & $.18^{* *}$ & $.15^{\circ}$ & .07 & -.02 & $-.15^{\circ}$ & $.71^{* *}$ & & \\
\hline 9. Conscienticusness & .83 & $.33^{=m}$ & $.24=*$ & $.27 * *$ & $.13^{*}$ & .05 & .05 & $.24^{* *}$ & $.18 \geqslant *$ & \\
\hline 10. Emotional stability & 83 & $-.17 *$ & -.12 & -.06 & $-.17^{*}$ & $-.32 \%$ & $-.33^{*}$ & .04 & .04 & -.06 \\
\hline
\end{tabular}

$* p<.05 . * 0<0.005$

Table 3. Descriptive Statistics (Mean, Standard Deviation, and Minimum-Maximum) for Self-Care, Personal Model Beliefs, and Personality Measures for Men and Women

Table 3

Descriptive Statistics (Mean, Standard Deviation, and Minimum-Maximum) for Self-Care,

Personal Model Beliefs, and Personality Measures for Men and Women

\begin{tabular}{|c|c|c|c|c|c|c|}
\hline \multirow[b]{2}{*}{ Variable } & \multicolumn{2}{|c|}{ Mean } & \multicolumn{2}{|c|}{ Standard deviation } & \multicolumn{2}{|c|}{ Min-Max } \\
\hline & Men & Women & Men & Women & Men & Women \\
\hline Diet & 0.01 & 0.01 & 0.67 & 0.68 & $-1.5-1.4$ & $-2.6-1.6$ \\
\hline Exercise & 0.06 & -0.03 & 0.75 & 0,75 & $-1.6-1.3$ & $-1.6-1.3$ \\
\hline Blood glucose testing & 0.02 & -0.04 & 0.94 & 0.96 & $-1.8-0.9$ & $-1.8-0.9$ \\
\hline Insulin administration & 0.14 & -0.03 & 0.58 & 0.93 & $-1.6-0.5$ & $-4.5-1.2$ \\
\hline Perceived impact & 3.25 & 3.24 & 0.86 & 0.87 & $1,0-4.8$ & $1,0-5,0$ \\
\hline Perceived threat & 2.79 & 2.57 & 0.82 & 0.79 & $1.0-4,6$ & $1.0-5.0$ \\
\hline Effectiveness to control diabetes & 3.57 & 3.68 & 0.65 & 0.63 & $2.0-5.0$ & $1.8-5.0$ \\
\hline Effectiveness to prevent diabetes & 3.81 & 4.00 & 0.58 & 0.60 & $2.4-5.0$ & $1.4-5.0$ \\
\hline Conscientiousness & 3.72 & 3.84 & 0.31 & 0.63 & $2.5-5.0$ & $2.0-5,0$ \\
\hline Emotional stability & 2.96 & 3.35 & 0.74 & 0.73 & $1.5-4.8$ & 134.9 \\
\hline
\end{tabular}

Note. For men, $n=95$; for women, $n=243$. Min-Max = minimum-maximum. 
Figure 1. Models of association between conscientiousness, emotional stability, personal model beliefs, and self-care. A: Conscientiousness as an independent predictor of self-care. B: Conscientiousness moderates association between beliefs and self-care. C: Beliefs mediate association between conscientiousness and self-care.

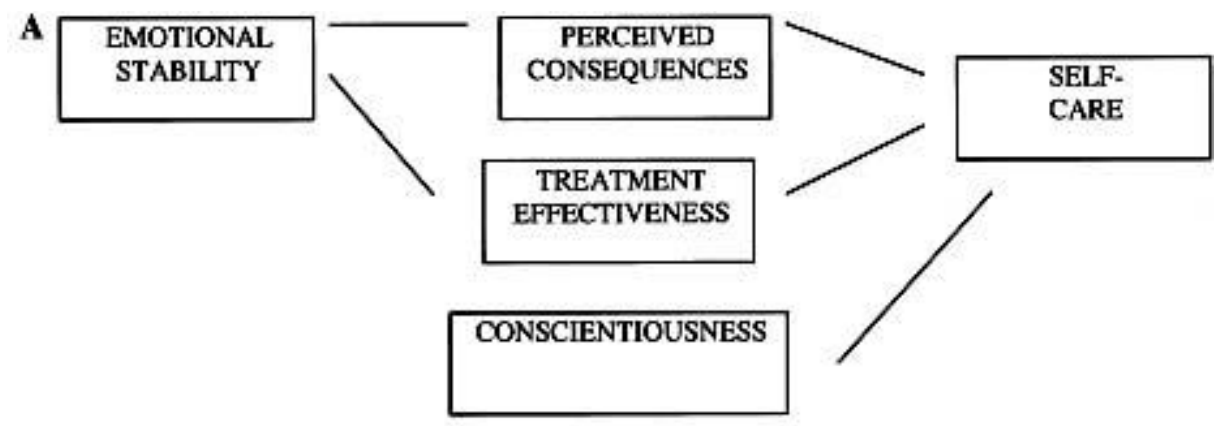

B

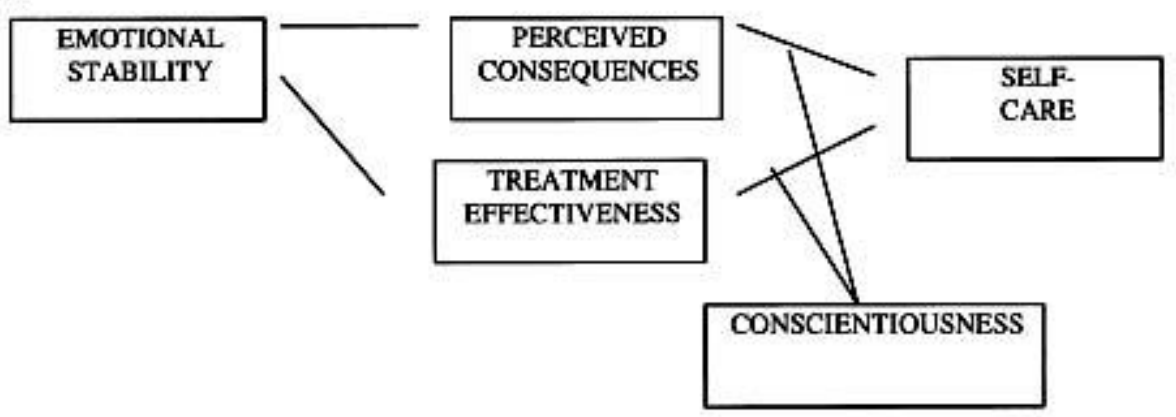

C

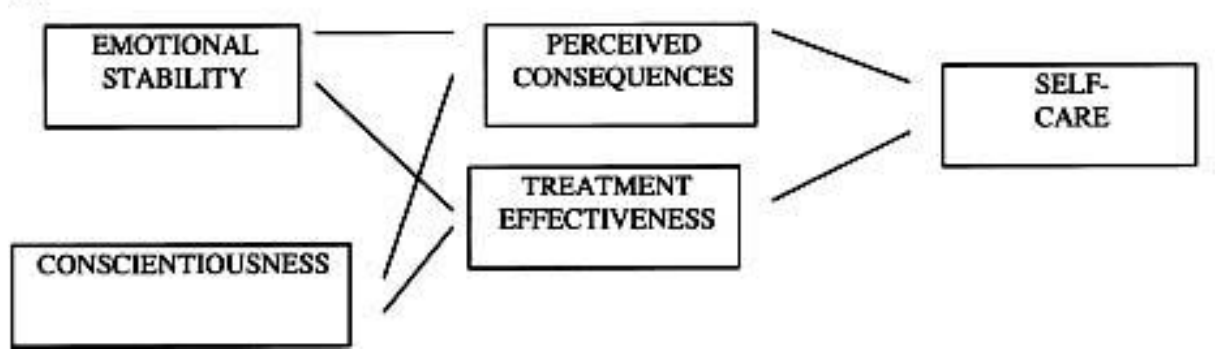


Figure 2. Structural equation model for conscientiousness, personal model beliefs, and selfcare.

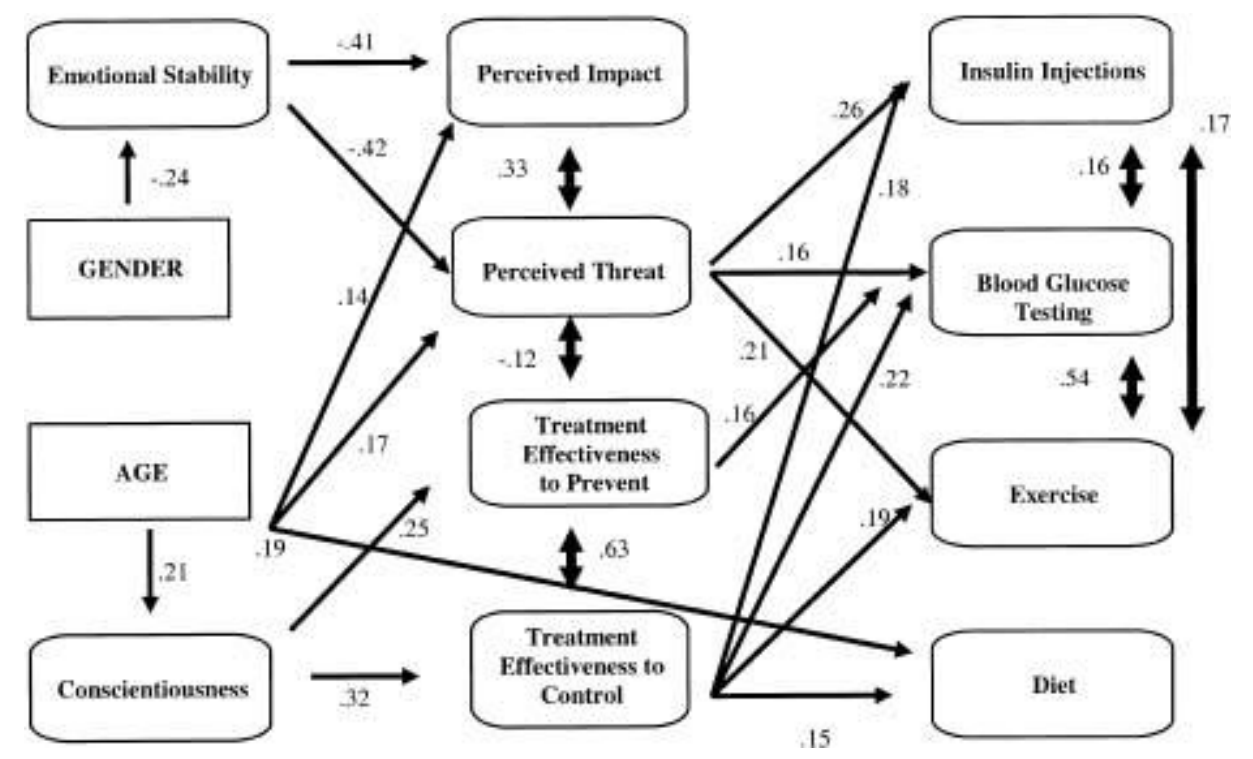

